\title{
Impedance spectroscopic study on hybrid phthalocyanine/lead sulfide nanocomposite film
}

Zahra Khozaee ${ }^{1}$, I. Chambrier ${ }^{2}$, Lydia X. Sosa Vargas ${ }^{2}$, Andrew N. Cammidge ${ }^{2}$, and Asim. K. Ray ${ }^{1,3}$

${ }^{1}$ Centre of Materials Research, Queen Mary, University of London, Mile End Road London, E1 4NS, UK

${ }^{2}$ School of Chemistry, University of East Anglia, Norwich Research Park, Norwich, NR4 7TJ, UK

\begin{abstract}
The frequency response of the AC conductivity of unique hybrid phthalocyanine/lead sulfide nanocomposite sandwiched between the indium tin oxide and aluminum electrodes is found to obey the universal power-law. The cryogenic study of the alternating current (AC) conduction reveals that the correlated barrier hopping $(\mathrm{CBH})$ model closely fits to the experimental data at temperatures below $240 \mathrm{~K}$. The parameters obtained by fitting the $\mathrm{CBH}$ model point out that the hopping process cannot take place directly between neighboring $\mathrm{PbS}$ QDs but involves the localised states within the phthalocyanine matrix.
\end{abstract}

Keywords: Nanocomposite, discotic, macrocyclic, octahexylphthalocyanine, quantum dots, CBH modeling. 


\section{Introduction}

Chemically and thermally stable, environmentally friendly non-toxic phthalocyanines (Pcs) are used in a range of applications from industrial pigments to photodynamic agents in cancer therapy and photosensitizers [1]. The conjugated cyclic $\pi$-systems of eighteen electrons in the macrocyclic ring gives rise to unique semiconducting properties, offering tremendous scope of developing field effect transistors, solar cells of power conversion efficiency and smart sensors for environmental pollution monitoring and biodetection in ppb level [2, 3]. Similarly, the size-dependent unique opto-electronic properties of crystalline semiconductor quantum dots (QDs) typically made of chalcogenides and phosphides of II to VI metals have attracted active research attention for their applications in nanoelectronics, optics and biological sciences $[4,5]$. In recent years, inorganic/organic hybrid nanocomposites incorporating semiconductor quantum dots into phthalocyanine matrix have attracted considerable interest in fundamental and applied research because of their diverse fields of applications in sensors [6,7], displays $[8,9]$, photovoltaics $[10,11]$ and photonic devices $[12,13]$.

A unique organic/inorganic nanocomposite of lead sulfide ( $\mathrm{PbS}$ ) quantum dots (QDs) embedded in non-peripherally octakis(hexyl) substituted metal-free phthalocyanine $\left(\mathrm{C}_{6} \mathrm{PcH}_{2}\right)$ has been prepared by a simple, one-step and low-cost method of $24 \mathrm{~h}$ long exposure of spin coated thin films of lead phthalocyanine derivative $\left(\mathrm{C}_{6} \mathrm{PbPc}\right)$ to the $\mathrm{H}_{2} \mathrm{~S}$ environment in a sealed container [14]. Transmission electron microscopic, X-Ray diffraction and UV-visible absorption studies have been performed on $\mathrm{C}_{6} \mathrm{PbPc}$ films, $\mathrm{C}_{6} \mathrm{PcH}_{2} / \mathrm{PbS}$ nanocomposite and pristine $\mathrm{C}_{6} \mathrm{PcH}_{2}$ films. The average $\mathrm{PbS}$ particle size is found to vary between $4.5 \mathrm{~nm}$ and 5.9 between obtained from TEM and XRD respectively. These values are much smaller than Bohr excitation radius of $18 \mathrm{~nm}$, indicating the formation of $\mathrm{PbS}$ quantum dots. Also, the value of $1.95 \mathrm{eV}$ is estimated for the band gap of $\mathrm{PbS}$ quantum dots. This is much larger than the bulk band gap of $0.41 \mathrm{eV}$ due to the quantum confinement [15]

In this work the frequency response of the conductivity of $\mathrm{C}_{6} \mathrm{PcH}_{2} / \mathrm{PbS}$ nanocomposite sandwiched between the ITO and Al electrodes is investigated by applying an AC signal with the amplitude of $0.1 \mathrm{~V}$ and sweeping frequency from $100 \mathrm{~Hz}$ to $1 \mathrm{MHz}$ at temperatures ranging from $100 \mathrm{~K}$ to $293 \mathrm{~K}$. In order to study the role of $\mathrm{PbS}$ quantum dots in the electrical properties of the nanocomposite device, the electrical properties of the pure matrix, $\mathrm{C}_{6} \mathrm{PcH}_{2}$, have been also investigated in the identical structural device and under the same ambient conditions. This dielectric relaxation spectroscopy which involves the examination of $\mathrm{AC}$ electrical response 
over a wide frequency range provides information on the conductivity of thin films of wide range materials in terms of structural homogeneity and stability considering relative contribution of grain, grain boundary and defect states [16].

\section{Experimental}

$\mathrm{C}_{6} \mathrm{PbPc}$ and pristine $\mathrm{C}_{6} \mathrm{PcH}_{2}$ compounds, the synthesis of which was reported earlier, were used in this investigation [17]. Spin coated films of both compounds were deposited onto ultrasonically cleaned substrates from spreading solutions in toluene, concentration of 10 $\mathrm{mg} / \mathrm{ml}$, using a KW-4A spin-coater (Chemat Technology Inc.) operating at $1000 \mathrm{rpm}$ for 30s. Under these conditions, deposited films were found to be approximately $130 \mathrm{~nm}$ thick. Following the observations described before [14], the nanocomposite $\mathrm{C}_{6} \mathrm{PcH}_{2} / \mathrm{PbS} / \mathrm{films}$ were produced through a $24 \mathrm{~h}$ exposure of spin-coated films of $\mathrm{C}_{6} \mathrm{PbPc}$ to a hydrogen sulphide $\left(\mathrm{H}_{2} \mathrm{~S}\right)$ environment in a sealed container. Under these conditions, the lead ion is removed and $\mathrm{PbS}$ nanoparticles are formed within a film of $\mathrm{C}_{6} \mathrm{PcH}_{2}$ as depicted in Figure 1(a).

The frequency response of the single-layered nanocomposite film sandwiched between an indium tin oxide (ITO) substrate and a $60 \mathrm{~nm}$ thick evaporated aluminium (Al) counter electrode, as shown in Figure 1(b), was obtained using Autolab potentiostat PGSTAT30. The experiment was run by applying an AC signal with amplitude of $0.1 \mathrm{~V}$ by sweeping frequency from $100 \mathrm{~Hz}$ to $1 \mathrm{MHz}$ at different temperatures between $100 \mathrm{~K}$ and $293 \mathrm{~K}$.. The instrument returns the real and imaginary parts of the admittance. The effective area of the devices was estimated to be $4.5 \mathrm{~mm}^{2}$ from the overlap between top Al and the bottom ITO electrodes. A similar sandwich structure device was also fabricated using spun $\mathrm{C}_{6} \mathrm{PcH}_{2}$ films as the semiconducting active material.

A continuous-flow cryostat, made by Oxford Instruments, was employed to measure the temperature-dependency of the electrical characteristic of the devices. The cryostat operated on the dynamic mode by circulating nitrogen gas around the device holder. During the experiment, the cryogen liquid continuously transferred from a cryogen container to the cryostat through a transfer tube. The temperature was controlled using ITC503 temperature controller, made by Oxford Instruments, by which the temperature was measured via a 
temperature sensor near the device holder, while the rate of cryogen flow was controlled manually. The temperature range used in this study is between $293 \mathrm{~K}$ and $100 \mathrm{~K}$.

\section{Results and discussion}

The real and imaginary parts of admittance, $Y^{\prime}$ and $Y^{\prime \prime}$ respectively, are obtained by applying an alternating field to the sandwich structure devices, $Y=Y^{\prime}+i Y^{\prime \prime}$. Having known the geometry of the devices, the AC conductivity is then estimated from the real part of admittance which is known as conductance, $\sigma_{A C}=\frac{d}{A} Y^{\prime}$, where $d$ is the thickness of the films, $d^{P c}=100 \mathrm{~nm}$ and $d^{N C}=130 \mathrm{~nm}$, and $A$ is the effective area of the devices $A=4.5 \mathrm{~mm}^{2}$. The superscripts $\mathrm{Pc}$ and $\mathrm{NC}$ are used for identifying the quantities corresponding to $\mathrm{C}_{6} \mathrm{PcH}_{2}$ and $\mathrm{C}_{6} \mathrm{PcH}_{2} / \mathrm{PbS}$ nanocomposite, respectively. Figures 2(a) and 2(b) show the logarithmic plots of frequency-dependence of the conductivity $\sigma_{A C}(\omega, T)$ for $\mathrm{C}_{6} \mathrm{PcH}_{2} / \mathrm{PbS}$ and $\mathrm{C}_{6} \mathrm{H}_{2} \mathrm{Pc}$ devices, respectively. The AC conductivity $\sigma_{A C}$ of the nanocomposite $\mathrm{C}_{6} \mathrm{PcH}_{2} / \mathrm{PbS}$ film is found to be higher than that of $\mathrm{C}_{6} \mathrm{PcH}_{2}$ device by about two orders of magnitude. However, the behaviour of $\mathrm{AC}$ conductivity with respect to frequency $f\left(=\frac{\omega}{2 \pi}\right)$ and temperature $T$ are almost similar for both devices. Significant increase in $\sigma_{A C}$ has been reported for optimized polyaniline-nickel ferrite nanocomposite over polyaniline due to the formation of $p$-n type heterojunction at the interface between n-type nickel ferrite and p-type polyaniline [18].

At a given temperature $T, \sigma_{A C}(\omega, T)$ increases monotonically by increasing frequency from $10^{2} \mathrm{~Hz}$ to $10^{6} \mathrm{~Hz} . \sigma_{A C}(\omega, T)$ is temperature-dependent and decreases by lowering temperature down to $T=100 \mathrm{~K}$. Similar frequency dispersion conductivity is reported for nanocomposite cadmium selenide sulphide/zinc sulphide quantum dots dispersed on 4-pentyl-4-cyanobiphenyl nematic liquid crystalline material. [19]. However, in disorder systems with lack of band-type conduction, the AC conductivity $\sigma_{A C}$ is frequency-dependent, universally observed to follow the universal power-law equation [20]:

$$
\sigma_{A C}(\omega, T)=\sigma_{D C}(T)+A \omega^{s}
$$

where $\sigma_{D C}(T)$ is the DC conductivity when $\omega \rightarrow 0, A$ is prefactor and $s$ is the frequency exponent. Both $A$ and $s$ are weakly dependent on temperature. The power-law frequencydependence is mostly observed in the frequency range from $\sim 1 \mathrm{~Hz}$ to $10 \mathrm{GHz}$ where the quantum and phonon effects become dominant [21].

The slope of $\sigma_{A C}(\omega, T)$ with respect to $f$ is plotted in Figure 2(c). The graphs can be 
approximately divided into low and high frequency regions corresponding respectively to the frequency below and above $10^{4} \mathrm{~Hz}$. The values of slope $s$ for $f<10^{4} \mathrm{~Hz}$ are less than unity, while they exceed unity at frequencies higher than $10^{4} \mathrm{~Hz}$. This type of behaviour is commonly reported for disordered semiconductors [22]. Values of slope below unity indicate that the frequency-dependence of the conductivity of both devices can be well described by the universal power-law in Equation (1) above. The frequency response of the materials has further been analysed.in order to determine the dominant $\mathrm{AC}$ conduction mechanism, The increase of $s$ with decreasing temperature $T$ is consistent with the correlated barrier hopping $(\mathrm{CBH})$ model in which the relaxation process is by hopping of charges between pair of localised states [23]. Similar conduction mechanism is reported for $8 \mathrm{~mm}$ in diameter and $1.1 \mathrm{~mm}$ in thickness of newly synthesized inorganic-organic tetrapropylammonium tetrachloferrate nanocomposite at temperature between $313 \mathrm{~K}$ and $388 \mathrm{~K}$ over the frequency range from $10^{3} \mathrm{~Hz}$ to $10^{7} \mathrm{~Hz}$ [24]. The consistency of the $\mathrm{CBH}$ model for both $\sigma_{A C}^{P C}$ and $\sigma_{A C}^{N C}$ is further investigated by fitting the following $\mathrm{CBH}$ equation (2) for both films.:

$$
\sigma_{A C}=\frac{\pi^{3}}{24} N^{2} \epsilon \varepsilon_{0} \omega R_{\omega}^{6}
$$

and

$$
R_{\omega}=\frac{e^{2}}{\pi \in \varepsilon_{0}\left(W_{m}+k T \ln \left(\omega \tau_{0}\right)\right)}
$$

Where $R_{\omega}, W_{m}$ and $N$ represent hopping distance, maximum barrier height and density of hopping sites, respectively. Values of these variables are found through the fitting process. The other parameters are characteristic relaxation time which is assumed to be equal to the inverse of phonon vibration frequency, $\tau_{0}=10^{-13} \mathrm{~s}$, The value of dielectric constant, $\varepsilon$, is found to be 5 and 6.2, respectively, for $\mathrm{C}_{6} \mathrm{PcH}_{2}$ and $\mathrm{C}_{6} \mathrm{PcH}_{2} / \mathrm{PbS}$ nanocomposite, Figures 3 and 4 depict the results of the fitting for both $\mathrm{C}_{6} \mathrm{H}_{2} \mathrm{Pc}$ and the $\mathrm{C}_{6} \mathrm{H}_{2} \mathrm{Pc} / \mathrm{PbS}$ nanocomposite, respectively. The Arrhenius plot of the temperature-dependence of the AC conductivity in the form of $\sigma_{A C}$ vs. 1000/T), and the temperature-dependence of the exponent $s$ ( $S$ vs. $T$ ). The theoretical values of $s$ are calculated using Equation [2] 


$$
S_{C B H}(\omega, T)=1-\frac{6 k T}{W_{m}+k T l\left(\omega \tau_{0}\right)}
$$

The typical values are calculated at the fixed frequency of $10^{3} \mathrm{~Hz}$. The frequency dispersion of $s$ is very weak and negligible (lower than \%1 and \%1.3 for the obtained values of $W_{m}^{P C}$ and $W_{m}^{N C}$, respectively. It is to be noted from the fitting results that the $\mathrm{CBH}$ model is well fitted to the $\mathrm{AC}$ conductivity of both $\mathrm{C}_{6} \mathrm{PcH}_{2}$ and the $\mathrm{C}_{6} \mathrm{PcH}_{2} / \mathrm{PbS}$ samples at temperatures lower than $240 \mathrm{~K}$. For higher temperatures ( $T>240)$, the temperature-dependence of $\sigma_{A C}^{P C}$ and $\sigma_{A C}^{N C}$ is much stronger than the prediction from the $\mathrm{CBH}$ model.

The obtained values of $W_{m}$ and $N$ for both materials have been listed in Table 1 . The value of $W_{m}$ for the $\mathrm{C}_{6} \mathrm{PcH}_{2} / \mathrm{PbS}$ nanocomposite is smaller than that of the $\mathrm{C}_{6} \mathrm{PcH}_{2}$ while $N$ of the $\mathrm{C}_{6} \mathrm{PcH}_{2} / \mathrm{PbS}$ nanocomposite is larger than that of $\mathrm{C}_{6} \mathrm{PcH}_{2}$. by a factor of 6 . These observations suggest that the incorporation of the $\mathrm{PbS}$ QDs in $\mathrm{C}_{6} \mathrm{PcH}_{2}$ introduces new hopping sites with smaller binding energy, which dominantly contribute to the AC conduction of the nanocomposite, at least for $T<240 \mathrm{~K}$. Some possible sources of the new hopping sites can be morphological defects introduced to the matrix by inclusion of the PbS QDs or probable surface states and dangling bonds of the PbS QDs. Using the relation of $R_{\omega}$, the most probable hopping length in the nanocomposite is found to be $0.69 \pm 0.1 \mathrm{~nm}$.

The average size of nanoparticles $D$ and the volume fraction of nanoparticles in this hybrid were previously found to be $4.5 \mathrm{~nm}$ and $3 \%$, respectively [15]. Having known these values, the average distance between centers of two neighboring particles, $\widetilde{r}$, is calculated to be $\sim 11.7$ $\mathrm{nm}$ using equation in the form [25]:.

$$
\tilde{r}=\left(6 x / D^{3} \pi\right)^{-1 / 3}
$$


The hopping length $\tilde{r}$ in the nanocomposite is found to $0.69 \pm 0.1 \mathrm{~nm}$, which is much smaller than the distance between the PbS QDs in the nanocomposite, $\sim 11.7 \mathrm{~nm}$, indicating there may not be any direct hopping between two neighboring QDs.

The relative dielectric constant of the device is calculated using the expression $\varepsilon=\frac{d}{\varepsilon_{0} A} \frac{Y^{\prime \prime}}{\omega}[26]$. Figure 5 shows the frequency-dependence of the dielectric constant of $\mathrm{C}_{6} \mathrm{PcH}_{2} / \mathrm{PbS}$ and $\mathrm{C}_{6} \mathrm{PcH}_{2}$ and $\mathrm{C}_{6} \mathrm{PcH}_{2} / \mathrm{PbS}$ nanocomposite device. The nanocomposite exhibits stronger frequency dispersion compared to the pure matrix. This may be referred to the presence of interfacial polarisation at the interface between the $\mathrm{PbS}$ QDs and the matrix. The interfacial polarisation typically observed in heterojunction systems when the polarisation abilities of the phases are different, and it is mainly dominant at low frequency region [27]. The frequencyand temperature-dependence of $\varepsilon$ in both devices are getting weak by decreasing temperature and become almost disappeared at around $200 \mathrm{~K}$. The value of dielectric constant at very high frequencies, near optical frequencies, corresponds only to electronic polarisation of materials (displacement of electrons around nuclei) rather than any other polarisation such as interfacial, dipole orientational and/or ionic polarisation. Lowering temperature can also reduce the effect of the orientational polarisation. Accordingly, the high-frequency dielectric constant can be determined from the saturation value of $\varepsilon$ at very high-frequency and low temperature. The dielectric constant of the materials at the highest attained frequency and lowest temperature is found to be $\sim 5$ and 6.2 corresponding to $\mathrm{C}_{6} \mathrm{PcH}_{2}$ and the $\mathrm{C}_{6} \mathrm{PcH}_{2} / \mathrm{PbS}$ nanocomposite, respectively.

\section{Conclusion}

The AC conductivity of the nanocomposite is frequency-dependent following the universal power-law in frequency ranging from $100 \mathrm{~Hz}$ to $1 \mathrm{MHz}$. The charge relaxation is found to occur by hopping between localised states mainly at $T<240 \mathrm{~K}$. By fitting data to the CBH model, the hopping length was found to be $0.69 \pm 0.1 \mathrm{~nm}$ which is much smaller than the 
estimated distance between the $\mathrm{PbS}$ QDs, $\sim 11.7 \mathrm{~nm}$, indicating that the direct hopping between the neighbouring QDs is very unlikely but it involves the localised states within the matrix.

\section{References}

1. C. G. Claessens, U. Hahn and T. Torres, Phthalocyanines: From outstanding electronic properties to emerging applications. Chem. Rec. 8(2) (2008) 75-97.

2. J. Chen, C. J. Zhu, Y. Xu, P. W. Zhang and T. X. Liang, Advances in Phthalocyanine Compounds and their Photochemical and Electrochemical Properties. Curr. Org. Chem. 22(5) (2018) 485-504

3. D Woehrle, G. Schnurpfeil, S.G. Makarov, A. Kazarin and O. N. Suvorova Practical Applications of Phthalocyanines - from Dyes and Pigments to Materials for Optical, Electronic and Photo-electronic Devices. Macroheterocyles 5(3) (2012) 191-202.

4. S. Chand, N. Thakur, S. C. Katyal, P. B. Barman, V. Sharma and P. Sharma, Recent developments on the synthesis, structural and optical properties of chalcogenide quantum dots. Sol. Energy Mater. Sol. Cells168 (2017) 183-200

5. H. Li, C. Jia, X. W. Meng and H. B. (Li, Chemical Synthesis and Applications of Colloidal Metal Phosphide Nanocrystals. 6 (2017) Article Number: 652

6. M. Bouvet, P. Gaudillat and J. M. Suisse, Phthalocyanine-based hybrid materials for chemosensing. J. Porphyr. Phthalocyanines 17(10) (2013) 913-919

7. L. Lu, Z. W. Zhu and X. Q. Hu, Hybrid nanocomposites modified on sensors and biosensors for the analysis of food functionality and safety. Trends Food Sci. Technol. 90 (2019) 100-110.

8. L. T. Yuan, L. Pan, K. Jia, Y. Y. Liu, Z. F. Huang, Y. M. Huang and X. B. Liu, Facile fabrication of white-emitting hybrid colloids and nanocomposite films using $\mathrm{CdSe} / \mathrm{CdS}$ quantum dots and zinc phthalocyanines as building blocks. Synth. Met. 218 (2016) 918.

9. S. Mandali, M. G. Iglesias, M. Ince, T. Torres and N. V. Tkachenko, Photoinduced Energy Transfer in ZnCdSeS Quantum Dot-Phthalocyanines Hybrids. ACS Omega 3(8) (2018) 10048-10057. 
10. A. Guchhait, S. Das, S. K . Ray and A. J. Pal, Photoinduced Hole-Transfer in Nanoparticle-Dye Hybrid Composites: A Route for Exciton Dissociation Leading to Photovoltaic Devices. Nanosci. Nanotechnol. Lett. 5(1) (2013) 13-18

11. P. Khurana, S. Thatai, N. B. Chaure, S. Mahamuni and S. Kulkarni, Solutionprocessed copper phthalocyanine-gold nanoparticle hybrid nanocomposite thin films. Thin Solid Films 565 (2014) 202-206

12. J. Wang, Y. J. Zhang, J. Q. and Z. Jiang, Enhanced fluorescence of tetrasulfonated zinc phthalocyanine by graphene quantum dots and its application in molecular sensing/imaging. Luminescence 32(4) (2017) 573-580.

13. K. Sanusi, S. Khene and T. Nyokong, Enhanced optical limiting performance in phthalocyanine-quantum dot nanocomposites by free-carrier absorption mechanism. Opt. Mater.37 (2014) 572-582.

14. A. V. Nabok, A. K. Ray, M. J. Cook, P. M. Burnham, Iwantono, H. Yanuar, M. Simmonds and T. V. Basova, Lead sulphide/phthalocyanine nanocomposite spun films, IEEE Trans. Nanotechnol. 3 (2004), no. 3, 388-394.

15. Z. Khozaee, L. Sosa-Vargas, A. N. Cammidge, M. J. Cook and A. K. Ray, Hybrid phthalocyanine/lead sulphide nanocomposite for bistable memory switches, Mater. Res. Express 2 (2015) 096305.

16. A. R. Long, Frequency-dependent loss in amorphous semiconductors, Advances in Physics 31(5), (1982) 553-637.

17. P. M. Burnham, I. Chambrier, D. L. Hughes, B. Isare, R. J. Poynter, A. K. Powell and M. J. Cook, Synthesis, lead lability and ring deformation of three 1,4,8,11,15,18,22,25octasubstituted phthalocyaninato lead derivatives, J. Porphyr. Phthalocyanines 10 (2006), 9-10, 1202-1211.

18. R. Megha, Y. T. Ravikiran, S. C. V. Kumari and S. Thomas, Influence of n-type nickel ferrite in enhancing the AC conductivity of optimized polyaniline-nickel ferrite nanocomposite. Appl. Phys. A-Mater. Sci. Process 123(4) 9(2017), Article Number: 245

19. G. Kocakulah, G. Onsal, K. Goksen, I. Ercan and O. Koysal, Concentration effect of Cadmium Selenide Sulphide/Zinc Sulphide quantum dots on electro-optic and dielectric properties in nematic liquid crystals composite Physica B 550 (2018), 4759.

20. A.K. Jonscher, Universal Relaxation Law (Chelsea Dielectric Press, London, 1996)

21. S. R. Elliott, Ac conduction in amorphous chalcogenid and pnictide semiconductors, Advances in Physics 36 (1987), no. 2, 135-218. 
22. A. K. Jonscher, Dielectric relaxation in solids, Chelsea Dielectrics Press Ltd, London, 1983.

23. A.R. Long Frequency-dependent loss in amorphous semiconductors Adv. Phys., 31 (1982), 553-637,

24. K. Ben Brahim, M. Ben Gzaiel, A. Oueslati, F. Hlel and M. Gargouri, Synthesis, structural characterization and electrical conduction mechanism of the new organicinorganic complex: ([(C3H7)(4)N]FeCl4) Mater. Res. Bull. 118 (2019), Article Number: 110505 .

25. K. R. Choudhury, M. Samoc, A. Patra and P. N. Prasad, Charge carrier transport in poly(n-vinylcarbazodle): Cds quantum dot hybrid nanocomposite, J. Phys. Chem. B 108 (2004), no. 5, 1556-1562.

26. R. D. Gould, Structure and electrical conduction properties of phthalocyanine thin films, Coord. Chem. Rev. 156 (1996), 237-274.

27. S. O. Kasap, Principles of electronic materials and devices, McGraw-Hill, 2006.

C. Tyagi and, A. Devi, Effect of Hybrid Organic-Inorganic Nanocomposite on Impedance Spectroscopy, Electric Modulus and Photoluminescence Property for Photovoltaics Applications., J. Nanoelectron. Optoelectron. 14(1), (2019) 128-135

G. Liu, X. Yang, A. Bonnefont, Y. K. Lv, J. Chen, W. Y. Dan, Z. F. Chen, L. Ruhlmann, D. S. Wright, and C. Zhang, Conjugated hybrid films based on a new polyoxotitanate monomer, Chem. Commun.54(100), (2018) 14132-14135

2. Sanchez, C. Boissiere, S. Cassaignon, C. Chaneac, O. Durupthy, M. Faustini, D. Grosso, C. Laberty-Robert, L. Nicole, D. Portehault, F. Ribot, L. Rozes, and C. Sassoye, Molecular Engineering of Functional Inorganic and Hybrid Materials. Chem. Mat. 26(1) Special Issue: SI (2014), 221-238. 


\section{Figure captions}

Figure 1 (a) Non-peripherally substituted at 1,4,8,11,15,18,22,25-sites with Octakis(hexyl) phthalocyanines showing the chemical structures of the lead metallated derivative, $\left(\mathrm{C}_{6} \mathrm{PbPc}\right)$, and the metal-free analogue $\mathrm{C}_{6} \mathrm{H}_{2} \mathrm{Pc}$ within a scheme illustrating the formation of lead sulphide $(\mathrm{PbS})$ quantum dots, (b) Sandwich device structure of $\mathrm{C}_{6} \mathrm{PcH}_{2} / \mathrm{PbS}$ nanocomposite between ITO and $\mathrm{Al}$ electrodes. Taken from [7].

Figure 2. The frequency dispersion of $\mathrm{AC}$ conductivity $\boldsymbol{\sigma}_{A C}$ of (a) $\mathrm{C}_{6} \mathrm{PcH}_{2}$ and (b) $\mathrm{C}_{6} \mathrm{PcH}_{2} / \mathrm{PbS}$ at different temperatures, and (c) the dependence of frequency exponent $\boldsymbol{s}$ on temperature $\boldsymbol{T}$. The error of the listed values is not more than $2 \%$.

Figure 3. Fitting the $\mathrm{CBH}$ model to the experimental data of $\mathrm{C}_{6} \mathrm{PcH}_{2}$ device presenting by (a) temperature-dependence of the $\sigma_{A C}$ conductivity (b) temperature-dependence of exponent $s$.

Figure 4. Fitting the $\mathrm{CBH}$ model to the experimental data of $\mathrm{C}_{6} \mathrm{PcH}_{2} / \mathrm{PbS}$ device presenting by (a) temperature-dependence of the AC conductivity (b) temperaturedependence of exponent $s$.

Figure 5. Frequency dispersion of dielectric constant of (a) $\mathrm{C}_{6} \mathrm{PcH}_{2}$ and (b) $\mathrm{C}_{6} \mathrm{PcH}_{2} / \mathrm{PbS}$ device.neighboring QDs. 
Table 1. Parameters applied for fitting the $\mathrm{CBH}$ model to the $\mathrm{AC}$ conductivity of the materials.

\begin{tabular}{lcccc}
\hline & $W_{m}(\mathrm{eV})$ & $N\left(\mathrm{~m}^{-3}\right)$ & $\varepsilon$ & $\tau_{0}(\mathrm{~s})$ \\
\hline $\mathrm{C}_{6} \mathrm{H}_{2} \mathrm{Pc}$ & 2 & $7.3 \times 10^{25}$ & 5 & $10^{-13}$ \\
$\mathrm{C}_{6} \mathrm{H}_{2} \mathrm{Pc} / \mathrm{PbS}$ & 1.8 & $4.5 \times 10^{26}$ & 6.2 & $10^{-13}$ \\
\hline
\end{tabular}




\begin{tabular}{|c|c|c|c|c|}
\hline \multirow{2}{*}{$\begin{array}{c}\text { Temperature } \\
(\mathrm{K})\end{array}$} & \multicolumn{4}{|c|}{$\mathrm{s}=\partial \sigma_{a c} / \partial f$} \\
\cline { 2 - 5 } & \multicolumn{2}{|c|}{$\mathrm{f} \leq 10^{4} \mathrm{~Hz}$} & \multicolumn{2}{c|}{$\mathrm{f}>10^{4} \mathrm{~Hz}$} \\
\cline { 2 - 5 } & $\mathrm{C}_{6} \mathrm{H}_{2} \mathrm{Pc}$ & $\mathrm{C}_{6} \mathrm{H}_{2} \mathrm{Pc} / \mathrm{PbS}$ & $\mathrm{C}_{6} \mathrm{H}_{2} \mathrm{Pc}$ & $\mathrm{C}_{6} \mathrm{H}_{2} \mathrm{Pc} / \mathrm{PbS}$ \\
\hline 293 & 0.85 & 0.67 & 1.74 & 1.65 \\
\hline 285 & 0.86 & --- & 1.78 & --- \\
\hline 265 & 0.89 & 0.83 & 1.96 & 1.78 \\
\hline 240 & 0.93 & 0.90 & 1.95 & 1.89 \\
\hline 200 & 0.96 & 0.94 & 1.96 & 1.95 \\
\hline 150 & 0.99 & 0.98 & 1.97 & 1.96 \\
\hline 100 & 1.02 & 0.99 & 1.97 & 1.96 \\
\hline
\end{tabular}

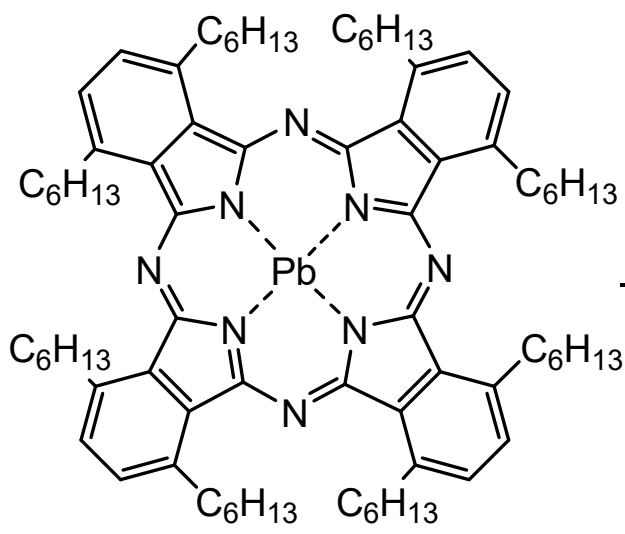

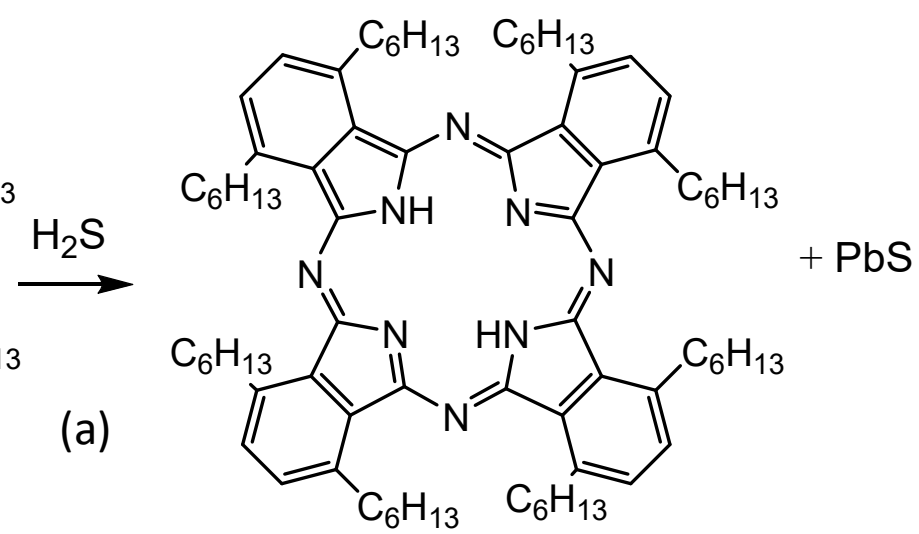

$6 \mathrm{PcPb}$

$6 \mathrm{PcH} 2$

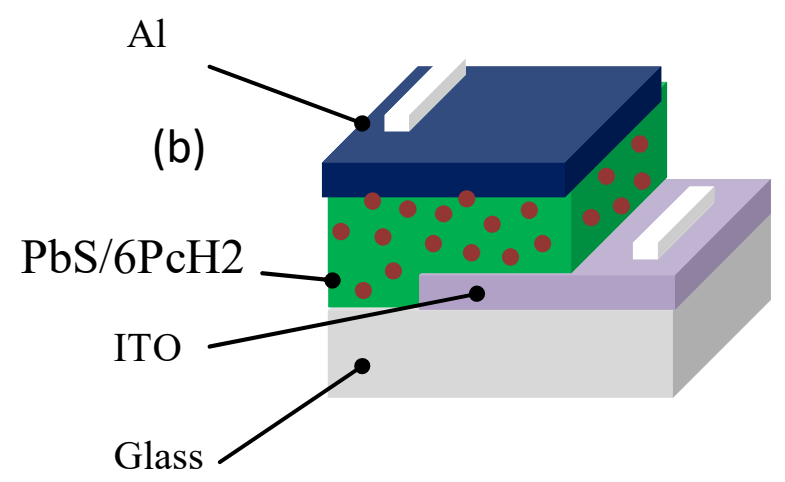

Figure 1 (a) 1,4,8,11,15,18,22,25-Octakis(hexyl) substituted phthalocyanines showing the chemical structures of the lead metallated derivative, $(6 \mathrm{PcPb})$, and the metal-free analogue $6 \mathrm{PcH} 2$ within a scheme illustrating the formation of lead sulphide (PbS) nanoparticles, (b) Sandwich device structure of $6 \mathrm{PcH} 2 / \mathrm{PbS}$ nanocomposite between ITO and Al electrodes. 

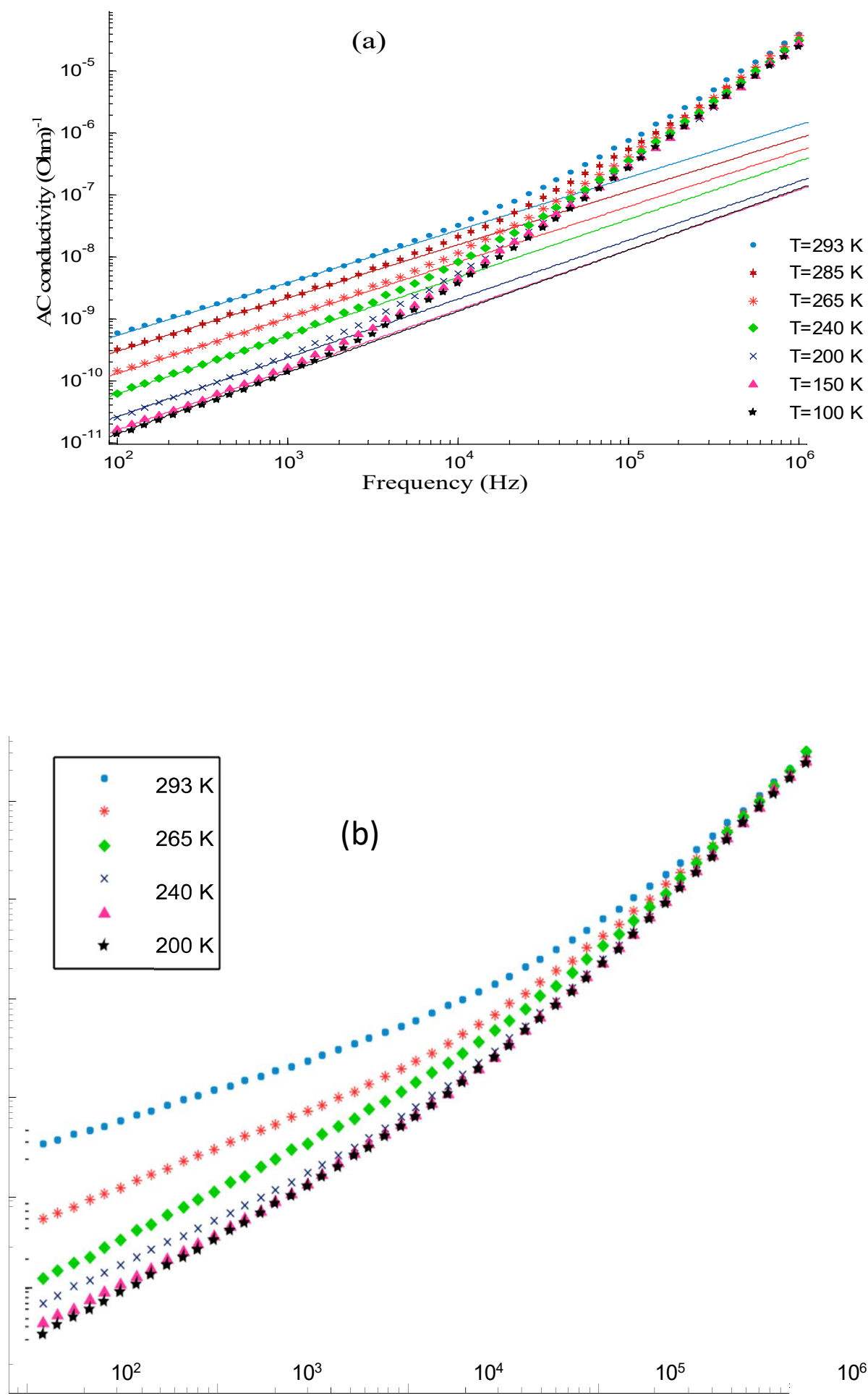

$10^{6}$

Frequency $(\mathrm{Hz})$ 


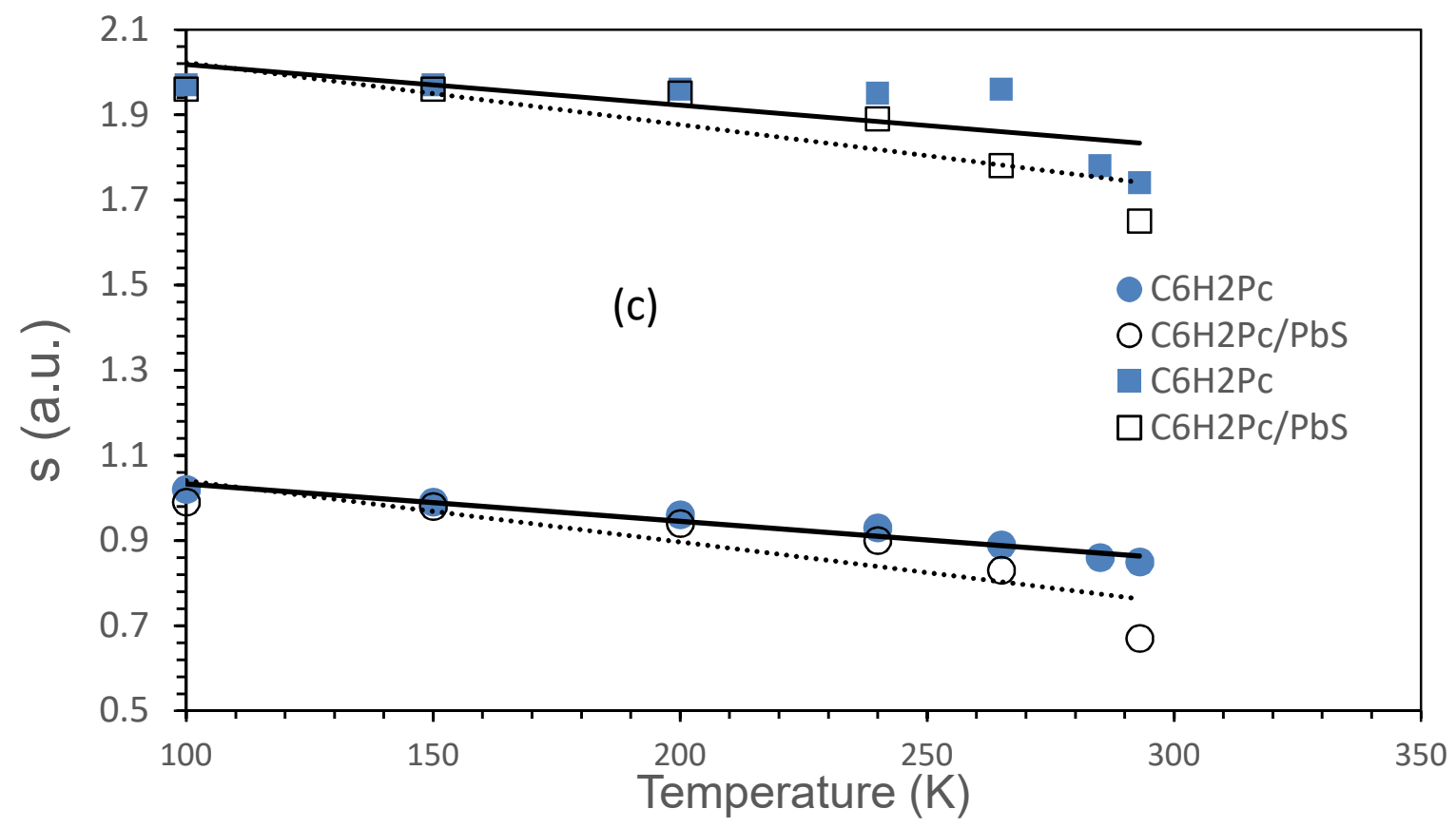

Figure 2. The frequency dispersion of AC conductivity $\boldsymbol{\sigma}_{A C}$ of (a) $6 \mathrm{PcH} 2$ and (b) $6 \mathrm{PcH} 2 / \mathrm{PbS}$ at different temperatures, and (c) the dependence of frequency exponent $\boldsymbol{s}$ on temperature $\boldsymbol{T}$ (square and circle symbols for f $\leq 10^{4} \mathbf{H z}$ and $\mathbf{f}>\mathbf{1 0}^{4} \mathbf{H z}$, respectively) The error of the listed values is not more than $2 \%$. 

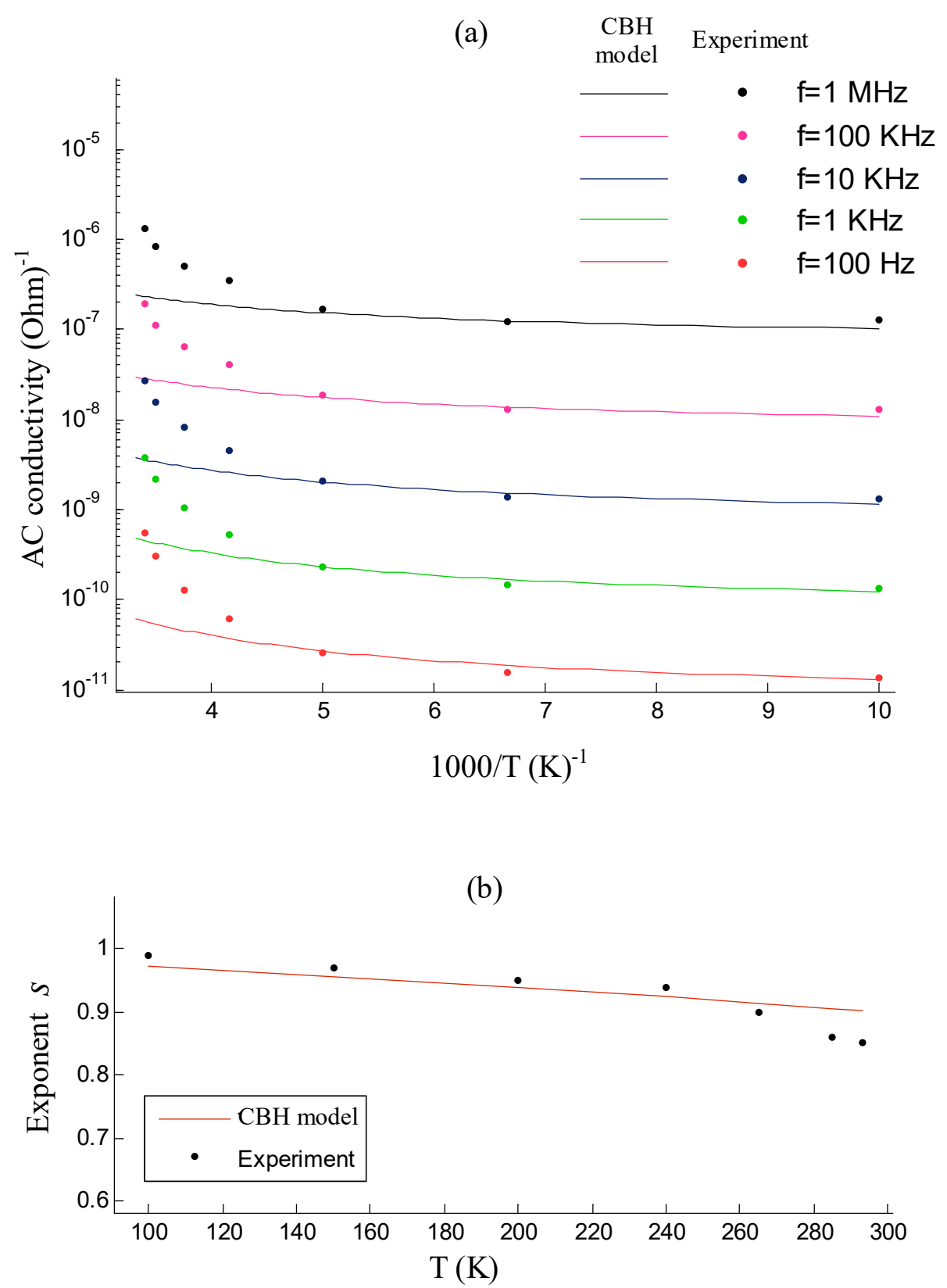

Figure 3. Fitting the $\mathrm{CBH}$ model to the experimental data of $6 \mathrm{PcH} 2$ device presenting by (a) temperature-dependence of the AC conductivity (b) temperature-dependence of exponent $s$. 


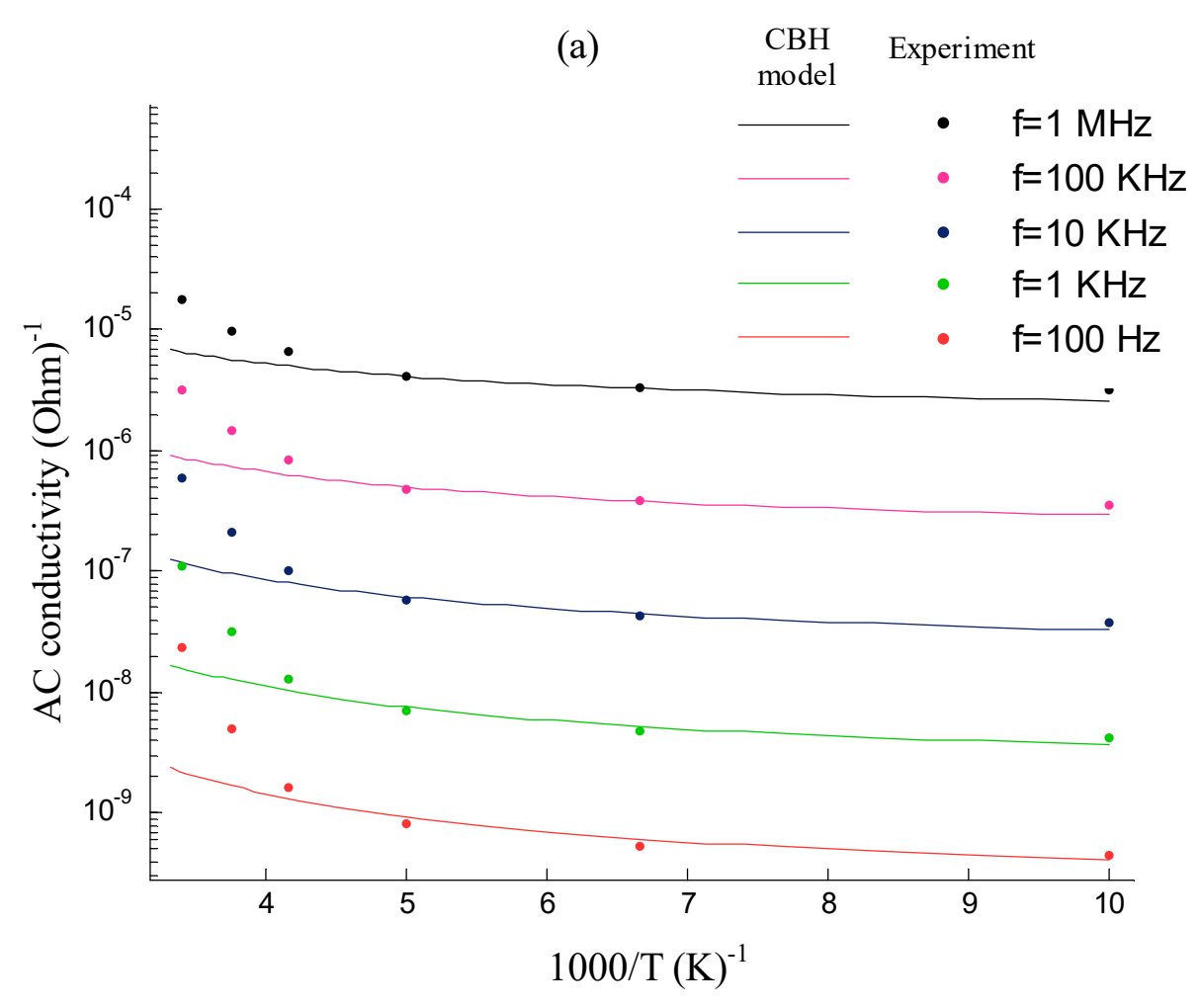

(b)

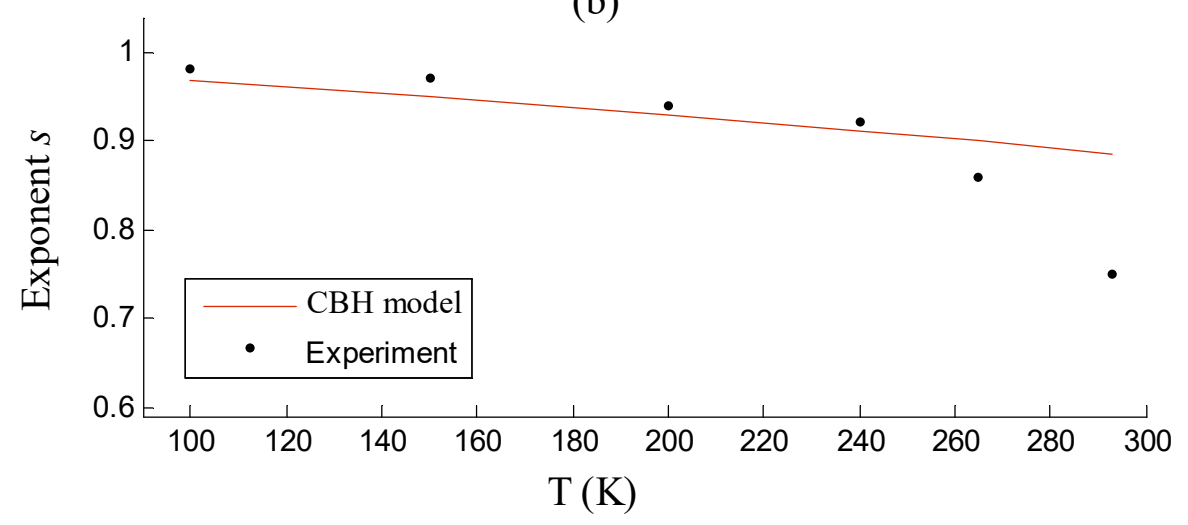

Figure 4. Fitting the $\mathrm{CBH}$ model to the experimental data of $6 \mathrm{PcH} 2 / \mathrm{PbS}$ device presenting by (a) temperature-dependence of the AC conductivity (b) temperaturedependence of exponent $s$. 

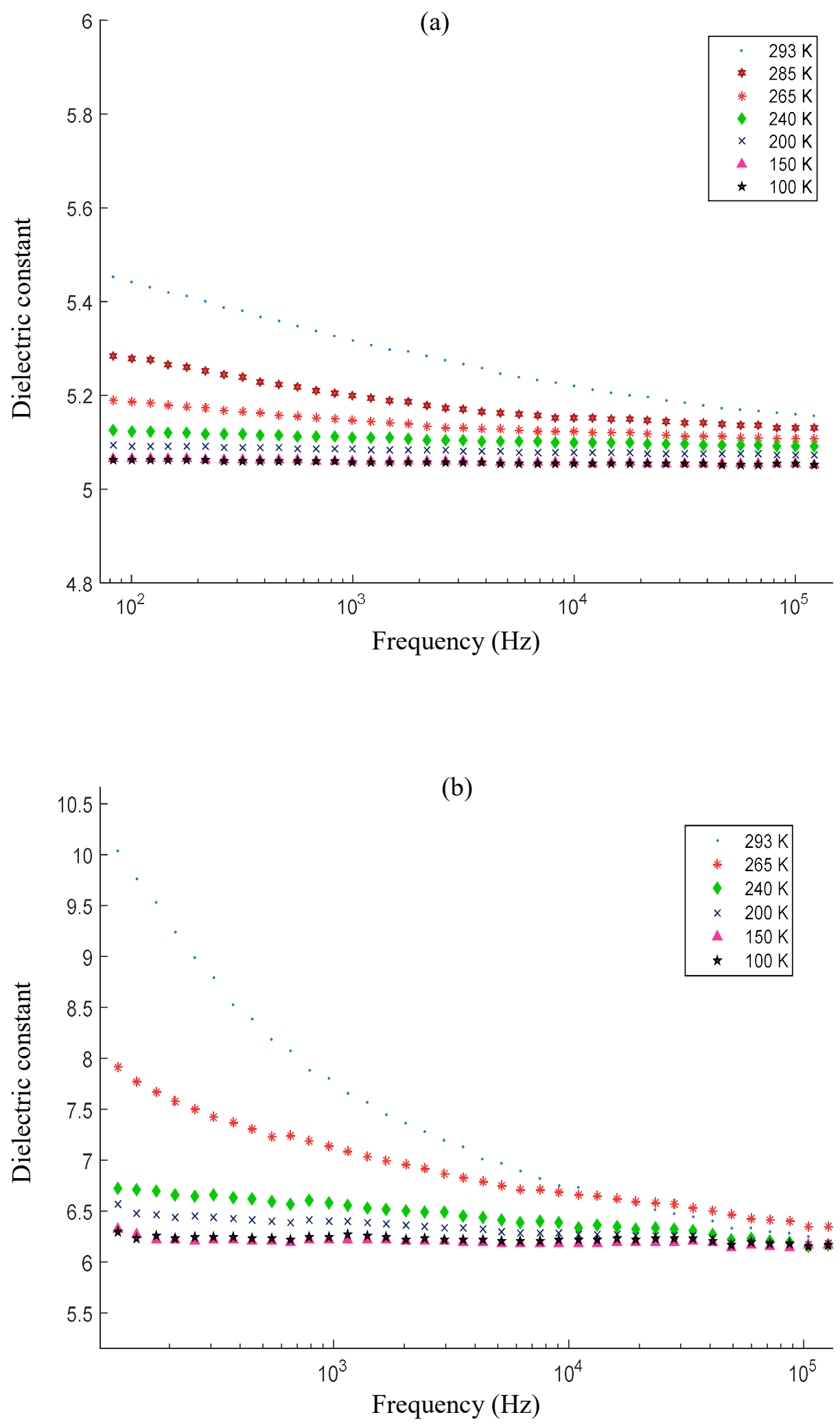

Figure 5. Frequency dispersion of dielectric constant of (a) $6 \mathrm{PcH} 2$ and (b) $6 \mathrm{PcH} 2 / \mathrm{PbS}$ device. 